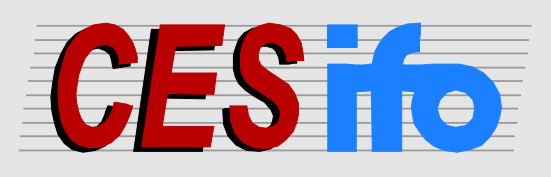

\title{
Working
}

Papers

www.cesifo.org/wp

\section{Hanging Together or Being Hung Separately: The Strategic Power of Coalitions where Bargaining Occurs with Incomplete Information}

\author{
Kai A. Konrad \\ Thomas R. Cusack
}

CESIFO WORKING PAPER NO. 4071

CATEGORY 2: Public CHOICE

JANUARY 2013

\footnotetext{
An electronic version of the paper may be downloaded

- from the SSRN website: Www.SSRN.com

- from the RePEc website: $\quad$ www.RePEc.org

- from the CESifo website: www.CESifo-group.org/wp
}

\section{CESifo}




\title{
Hanging Together or Being Hung Separately: The Strategic Power of Coalitions where Bargaining Occurs with Incomplete Information
}

\begin{abstract}
What is the strategic role of membership in an intergovernmental group with unanimity requirements if the group negotiates with an external player in a setting with incomplete information? Being in such a group has a strategic effect compared to negotiating as a standalone player and reduces the demands of the outside player: being in a group lends additional bargaining power. Negotiating as a group may also cause more inefficiencies due to bargaining failure, and this may harm also the intergovernmental group. We uncover the role of preference alignment and preference independence between members of the coalition group for equilibrium payoffs and welfare effects. In this analysis we also distinguishing between coalition groups with and without side payments. Overall, coalition groups tend to perform well for the members of the coalition group in comparison to fully decentralized negotiations, particularly if the objectives of the members of the coalition group are not always perfectly aligned.
\end{abstract}

JEL-Code: F510, F530, F590.

Keywords: bargaining, incomplete information, coalitions, groups, strategic bargaining power.

Kai A. Konrad
Max Planck Institute for Tax Law
and Public Finance
Munich / Germany
kai.konrad@tax.mpg.de

Kai A. Konrad and Public Finance

kai.konrad@tax.mpg.de 


\section{Introduction}

Joint decision making is a concept with a considerable history of thought in political science and economics. Scharpf's (1988) seminal paper set the stage for much of the discussion that followed. He argued that the unanimity requirement for policy reform may cause inefficient policy outcomes and highlighted the role of intergovernmental bargaining. Scharpf's analysis and much of the discussion it generated focuses on the quality of decision making inside a group of governments if they can depart from the status-quo only by unanimity. We do not focus on the quality of internal decision processes inside an intergovernmental group. Rather, we consider the implications of the structure of these internal decision processes for negotiations by this intergovernmental group with non-members of the group.

More specifically, we discuss three different types of decision structures of the intergovernmental group consisting of two players ( $A$ and $B)$ with respect to proposals made by a non-member of the group, which is player $S$. First, one decision structure makes both players $A$ and $B$ negotiate as stand-alone players. Player $S$ negotiates independently with each of them. Second, for a low degree of integration, $A$ and $B$ negotiate as an intergovernmental group: the offers made by $S$ are either both accepted by unanimous consensus, or both rejected. We call this type of intergovernmental group a coalition with weak ties. Third, players $A$ and $B$ may be more fully integrated and maximize their joint welfare when replying to $S$ 's offer and where they can make side payments to each other. We call this type of intergovernmental group a coalition with strong ties.

Negotiations by the set of member countries of the European Union with third parties in different policy areas provide examples for the different negotiation structures. On some policy issues each member country acts as a stand-alone player. Tax policy is largely a matter of the individual nation states within Europe. Accordingly, tax treaties such as information exchange agreements or double taxation treaties are negotiated and enacted on a strictly bilateral basis between single member countries and the respec- 
tive non-member country. On some other issues the European Union is the official counterpart in negotiations with a third party, but the individual member countries may still have important veto rights. International climate negotiations may serve as an example for negotiations by the member states as a coalition. ${ }^{1}$ The negotiations and decisions about the financial rescue packages for Greece in May 2010, and future rescue decisions in the context of the European Fiscal Stabilization Fund or the European Stability Mechanism have also followed this pattern. Positive decisions on rescue packages required unanimous consensus. ${ }^{2}$

We formally model the role of coalitions in a bargaining framework with one-sided incomplete information. One player $S$ bargains with two other players $A$ and $B$. Player $S$ has two items to which he does not attribute any value. One of these items he offers to $A$, the other to $B$. This is done without knowledge of the buyers' willingness to pay for the items. He makes simultaneous take-it-or-leave-it offers. We compare the asking prices, $A$ 's and $B$ 's payoffs and overall efficiency in the equilibrium for three bargaining regimes. We identify two major effects of coalitions with weak or strong ties, compared to the stand-alone regime: a strategic effect and an efficiency effect. The unanimity requirement may induce player $S$ to reduce his demands in the negotiations - hence, the unanimity requirement may shift bargaining power away from the proposing player $S$ towards the members of the intergovernmental group. This is the strategic effect of acting as a coalition. The unanimity requirement also changes the set of possible asking prices that would be accepted by the players $A$ and $B$. The set of combinations of prices for which efficiency enhancing trade takes place is changed. This describes the efficiency effect. We determine the conditions

\footnotetext{
${ }^{1}$ Eppstein, Gerlach and Huser (2010, p.10), for instance, describe the difficulties and the internal debate among member states during the Copenhagen climate summit.

${ }^{2}$ Whether this type of coalition negotiations is closer to the case with side payments or to the case without side payments depends on the internal governance structure of the European Union. It may often be difficult to assess whether a given European program with redistribtional impact was, in fact, a compensation payment.
} 
for when the increased bargaining power can overcompensate the enhanced efficiency cost for the intergovernmental group and when this can yield a higher expected payoff for the intergovernmental group. The determinants are (i) the alignment or independence of the preferences of members of the intergovernmental group, (ii) the nature of incomplete information, (iii) the structure of intergovernmental decision making inside the coalition.

While we have in mind other governments negotiating with the intergovernmental group, similar issues of commitment power have been debated in the context of whether governments are able to commit to policies that are not time consistent. ${ }^{3}$

Consider, for example, papers dealing with the EU. Jupille (1999) focuses on the issue of unanimity versus majority voting inside the EU for international outcomes in which the EU is one of several players. While Jupille's focus on majority voting versus unanimity as well as the decision framework differ from ours, he does consider the importance of intergovernmental decision rules for the group's bargaining power and for the type of outcomes that can be expected to emerge. Meunier (2000) finds that the bargaining process between the EU and its partners in international negotiations is affected by the process of decision making inside the EU. She discusses veto power by each individual member and majority rule as one dimension, considerations of delegation of power to a common negotiator as a second dimension and the type of bargaining process as a third dimension, using specific cases of conflict between the US and the EU on matters of trade. Groenleer and van Schaik (2007) point to the importance of preference congruence among the member states of the EU for international negotiations.

There are papers that explore more general applications. For example a formal analysis of the power of veto players in a country for the state's

\footnotetext{
${ }^{3}$ An example is government debt, and the government's incentive to default on this debt rather than charge distortionary taxes to service this debt. Stasavage (2002), for instance, analyzes this incentive focusing on the historical example of England and the roles of multiple veto players and multiplicity of policy issues for achieving credibility.
} 
bargaining power in international interactions is found in Kroll and Shogren (2008). If some extreme groups in a country have veto power, this changes the set of agreeable Pareto improvements and generates strategic commitment power. Kroll and Shogren's analysis is one with perfect and complete information. Manzini and Mariotti (2009a) consider several heterogenous players negotiating with a single player under complete information. The two sides make alternating offers as in a non-cooperative bargaining game. The authors show that this bargaining game has an equilibrium outcome similar to one in which the coalition delegates bargaining to the member of the group who is most reluctant to accept an agreement. In a companion paper Manzini and Mariotti (2009b) show how incomplete information can modify this effect. Bond and Eraslan (2009) also consider a group negotiating with a single player, assuming that the group votes on this player's proposal and accepts outcomes either by majority voting or by a unanimity requirement. In their framework the members of the group only receive a signal about how beneficial a positive outcome is for them, and the voting rule is used as an information aggregation device, related to Condorcet's jury theorem, but with strategic voting as in Feddersen and Pesendorfer (1998).

In all these approaches the negotiations are about one single item, something that could be seen as a public good to the group. Our approach considers bargaining in which each of the group members could make a deal with the single player on an individual basis. We compare individual trade relations with group decisions (with and without intragroup transfers). We allow for some structure on the preference distribution within an alliance and exploit the role of this distribution, comparing individual decision making and two types of group decision making. Our analysis takes the governance structures as given and compares equilibrium outcomes for different structures. ${ }^{4}$

\footnotetext{
${ }^{4}$ The problems of formation of alliances has received considerable attention. An important example is Hyndman and Ray (2007) who analyse a very general and rich framework
} 
The model laid out in this paper has quite a number of features common to a broad set of collective political action problems. This is especially the case with respect to the multiple concept framework that Scharpf (2000) has proposed as being useful for interpreting and understanding the distinctive forms of governance within the European polity. Outside of interactive processes that exclusively involve mutual adjustment, Scharpf suggests that there is a hierarchical set of decision dynamics that mark this polity. At the bottom, and involving the lowest level of institutionalization, are intergovernmental negotiations; at the top is the supranational hierarchical mode, and in the middle is the joint-decision mode.

Negotiations in the intergovernmental mode are fully controlled by national governments, and the agreements these countries reach require the consent of all as does their implementation. In stark contrast, hierarchical direction completely centralizes competencies at the European level; they are exercised by supranational actors alone. In practice, these functions are performed by institutions such as the ECB which, under most circumstances, are insulated from the influence of any democratically accountable agents. Sandwiched between these two extremes is the joint-decision mode, often labelled the "joint-decision trap" (Scharpf, 1988) because of its putative tendency to produce inefficient outcomes that the parties are incapable of escaping. This arises from two related conditions: (1) the central or encompassing government policy decisions flow only from agreement of the constituent governments and (2) the agreement of the latter occurs only with unanimity or near-unanimity. Scharpf (1988) has argued that this mode has the tendency to generate inefficient outcomes and that its legitimacy is undermined because of the role of non-accountable supranational actors, such as the Commission, to the extent that they can "compel national governments to change their positions on politically salient issues" (2000, p. 19). Elsewhere (2006, pp. 850-1), however, he stresses the beneficial role that

with a dynamic process of coalitions being formed and altered as an equilibrium process, complemented by a process of negotiations. 
the Commission plays since it has the monopoly on legislative initiative thereby tremendously reducing the transaction costs involved in arriving at consensual policy solutions.

\section{The analytical framework}

Consider three players, $A, B$ and $S$. Player $S$ is the stand-alone player. This player is in possession of two items, $a$ and $b$. Player $A$ is interested in obtaining item $a$, and player $B$ is interested in obtaining item $b$. For concreteness, we may think of $A$ and $B$ as member countries of the European Union, and $S$ a non-member country, potentially a tax haven, which negotiates about bilateral information exchange agreements or about double tax treaties, but $a$ and $b$ can also be very different political concessions, political favors or other politically tradeable goods. We assume that player $S$ does not attribute any value to items $a$ and $b$ (which is essentially a normalization assumption), and that players $A$ and $B$ attribute values to receiving these goods that are equal to $v_{A} \in[0,1]$ and $v_{B} \in[0,1]$. We assume that these values are draws from a joint distribution with the cumulative distribution function $F_{A B}\left(v_{A}, v_{B}\right)$.

To allow for straightforward comparative static results on the distribution function, we consider the following two probability models for the joint distribution $F_{A B}\left(v_{A}, v_{B}\right)$ that allow us to describe a continuum between stochastic independence and perfect positive or negative correlation by a single variable $\beta \in[0,1)$ :

$$
F_{A B}\left(v_{A}, v_{B}\right)=(1-\beta)\left[Z\left(v_{A}\right) Z\left(v_{B}\right)\right]+\beta Z\left(\min \left\{v_{A}, v_{B}\right\}\right)
$$

for the case with positive correlation, and

$$
F_{A B}\left(v_{A}, v_{B}\right)=\left\{(1-\beta)\left[Z\left(v_{A}\right) Z\left(v_{B}\right)\right]+\beta \max \left\{\left(v_{A}+v_{B}-1\right), 0\right\}\right.
$$

for the case with negative correlation. Here, $Z(z)$ is the cumulative distribution function of a univariate random variable that is uniformly distributed 
on $[0,1]$, and $\beta \in[0,1)$. Independence applies if $v_{A}$ and $v_{B}$ are two statistically independent draws from the distribution $Z(z)$. This is the case in both (1) and (2) for $\beta=0$. Perfect positive correlation applies in (1) if a single draw occurs and $z=v_{A}=v_{B}$. Perfectly negative correlation occurs if there is a single draw, with $v_{A}=z$ and $v_{B}=1-z$. These are the limit cases in (1) and (2) for $\beta \rightarrow 1$. Positive $\beta$ smaller than 1 maps cases with some, but not perfect correlation. This set-up will allow us to make comparative static analysis via a change in $\beta .^{5}$

The interaction between players is described by a version of the bargaining framework with incomplete information that has been introduced by Harsanyi and Selten (1972) and developed further by Chatterjee and Samuelson (1983). Player $S$ can commit to make a take-it-or-leave-it offer. We will discuss several regimes. The regime and its rules are exogenously given, and the rules are common knowledge.

In the standalone regime player $S$ makes separate offers to $A$ and to $B$. These offers are described by the asking prices $p_{A} \in[0,1]$ and $p_{B} \in[0,1]$ : the compensation which $S$ demands for the delivery of the items $a$ and $b$ to $A$ and $B$, respectively. Considering the tax-haven example, we may think of $a$ and $b$ as concessions such as the willingness to answer information requests about account holders from countries $A$ and $B$, or bank secrecy rules, and $p_{A}$ and $p_{B}$ may be development aid or other valuable benefits. Players $A$ and $B$ then consider these offers and each of them decides separately whether to accept or reject the offer. This ends the game. The payoffs accruing from

\footnotetext{
${ }^{5}$ The probability model that generates (1) can be seen as a two-stage compound lottery: in a first stage it is randomly decided whether a single draw from $Z(z)$ is made and $z=v_{A}=v_{B}$, or whether two statistically independent draws of $v_{A}$ and $v_{B}$ are made. The probability for a single draw is $\beta$. In a second stage, $z$ is drawn (once or twice, respectively). This two-stage lottery generates $F_{A B}\left(v_{A}, v_{B}\right)$ as in (1) as a linear combination of two distributions of $\left(v_{A}, v_{B}\right)$, one with perfect correlation, and one with stochastic independence. The construction of (2) is analogous. But in this case, with probability $\beta$, the single draw of $z$ yields $v_{A}=z$ and $v_{B}=1-z$, and with probability $1-\beta$, the values $v_{A}$ and $v_{B}$ are statistically independent draws.
} 
these outcomes are $\pi_{S}=\theta_{A} p_{A}+\theta_{B} p_{B}$ for player $S$ and $\pi_{A}=\theta_{A}\left(v_{A}-p_{A}\right)$ and $\pi_{B}=\theta_{B}\left(v_{B}-p_{B}\right)$ for players $A$ and $B$, where $\theta_{A}$ and $\theta_{B}$ are indicator variables taking values 1 and 0 if the individual player accepts or rejects the offer.

In the coalition regime with weak ties, $A$ and $B$ are part of a specific type of collective decision framework as follows: player $S$ makes offers with asking prices $p_{A}$ and $p_{B}$. Then both $A$ and $B$ announce separately and simultaneously whether or not they are willing to accept the price offer. The offers $\left(p_{A}, p_{B}\right)$ are collectively rejected if at least one of the players announces rejection. Only if both players announce acceptance does trade take place; $A$ pays $p_{A}$ and receives $a, B$ pays $p_{B}$ and receives $b$. This regime maps, for instance, negotiations of the European Union with a non-member state. In these negotiations each member-state has a veto right. Whether or not a non-member country is admitted to the European free-trade area, or other changes of the European Treaty that regulate interaction with nonmember states, with veto rights of each member state, may serve as an illustration.

In the coalition regime with strong ties players $A$ and $B$ can make a more powerful commitment, involving an information exchange and side payments between $A$ and $B$ in the event of the offer being accepted. Player $S$ makes a joint offer $p_{A}+p_{B} \equiv p_{A B}$ which is the asking price requested by $S$ for the delivery of items $a$ to $A$ and $b$ to $B$. Now players $A$ and $B$ learn their reservation values $v_{A}$ and $v_{B}$ that each holds. This information transfer is part of the coalition set-up and is assumed to be completely truthful. ${ }^{6}$ Players $A$ and $B$ make a decision about accepting or rejecting player $S$ 's proposal. Players $A$ and $B$ apply an internal sharing rule such that, in case of acceptance, they arrange for side payments to share any possible surplus

\footnotetext{
${ }^{6}$ Bearce, Flanagen and Floros (2006) pointed out that information transfer is an essential element of the formation of alliances. Konrad (2012) used this insight in a conflict framework in which an information transfer about military capacity is the only aspect of the formation of alliances.
} 
$v_{A}+v_{B}-p_{A B}$ equally. If $A$ or $B$ objects to the deal, then no deal takes place. If both players announce acceptance, then the items are delivered and the coalition of $A$ and $B$ must jointly pay the price $p_{A B}$. This regime may also apply for negotiations of the European Union on some matters. Explicit side payments in the European Union are rare. However, the negotiations on the size and spending categories in the Multiannual Financial Framework of the European Union provides ample opportunities to agree on internal side payments.

In what follows, we solve these three games separately from each other. Then we compare the outcomes for the asking price and for the coalition players. Readers who are not interested in the formal analysis may jump to the comparison section directly; this section also offers some graphical explanation for the incentives that are at work.

\section{The stand-alone regime}

Consider first the stand-alone regime. Player $A$ knows his $v_{A}$ and receives an offer with asking price $p_{A}$. Player $A$ accepts this offer if $p_{A} \leq v_{A}$ and a deal as regards $a$ takes place. If $p_{A}>v_{A}$, then this offer is unfavorable for $A$, the player rejects and $a$ is not traded. Similarly for $B$. The decisions of $A$ and $B$ and the resulting trading outcomes are unrelated in this regime. Given this, player $S$ solves two separate, but identical problems for $A$ and $B$. Player $S$ chooses asking prices that are determined by

$$
p_{I}=\arg \max _{p}\{(1-Z(p)) p\} \text { for } I \in\{A, B\}
$$

Given the assumptions about the marginal distributions, the optimal choice is the same for both countries and is given by the solution to $(1-Z(p))-$ $Z^{\prime}(p) p=0$, which yields equilibrium prices and resulting ex-ante expected payoffs for $A, B$ and $S$ of

$$
p_{A}=p_{B}=\frac{1}{2} ; \pi_{A}=\pi_{B}=1 / 8 \text { and } \pi_{S}=1 / 2 .
$$




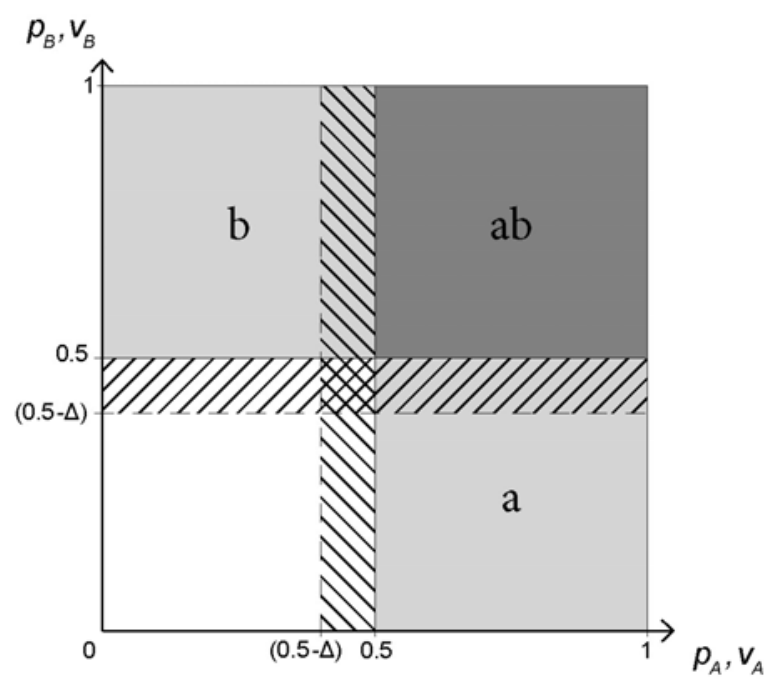

Figure 1: For the asking price $p_{A}=p_{B}=1 / 2$ both players accept if $\left(v_{A}, v_{B}\right)$ is inside the area ab, only player $A$ (resp. $B$ ) accepts if $\left(v_{A}, v_{B}\right)$ is inside a (resp. b). A reduction in the asking prices by $\Delta$ increases the areas in which acceptance takes place.

Figure 1 shows the range of possible combinations of $v_{A} \in[0,1]$ and $v_{B} \in$ $[0,1]$ and illustrates the equilibrium offers $p_{A}=p_{B}=1 / 2$ for the stand-alone regime for $\beta=0$ (stochastic independence).

At $p_{A}=p_{B}=1 / 2$ a reduction in $p_{A}$ and $p_{B}$ by $\Delta$ has a price effect and a quantity effect. It lowers the payment received by $S$ if trade takes place (price effect), but it increases the expected number of trades that take place by $2 \Delta$ (quantity effect). At the equilibrium asking price, the price and quantity effect of a small increase or decrease in the price just balance each other with respect to the payoff of $S$.

\section{A weak-ties coalition}

We turn next to the case of a weak-ties coalition. Let us first consider the decision of one player, say player $A$ for a given asking price $p_{A}$. If $p_{A}<v_{A}$ then player $A$ would like to make the deal, and if $p_{A}>v_{A}$, then $A$ is better- 
off if the deal does not take place. Indifference prevails if $p_{A}=v_{A}$. The optimal reply for player $A$ is not only a function of this comparison, but also of the expectation about the acceptance decision of player $B$. We assume that $v_{A}$ and $v_{B}$ remain fully private information to the players $A$ and $B$ in the weak-ties coalition and also that each player observes only the price requested from him. ${ }^{7}$

The acceptance choice of player $A$ can be conditional on $v_{A}$ and the price $p_{A}$ only, and player $S$ cannot observe an action or a signal that could make this player update the prior belief about player $A$ 's and player $B$ 's type. It is easy to confirm that a perfect Bayesian equilibrium is characterized by decisions as follows ${ }^{8}$ : Each player $A$ and $B$ accepts if and only if the price requested is not higher than the player's private valuation. Player $S$ anticipates this behavior and maximizes expected return $\left(p_{A}+p_{B}\right)\left[\operatorname{prob}\left(p_{A} \leq v_{A}\right.\right.$ and $\left.p_{B} \leq v_{B}\right)$ ], which can be expressed as

$$
\left(p_{A}+p_{B}\right)\left(1-p_{A}-p_{B}+F_{A B}\left(p_{A}, p_{B}\right)\right)
$$

The term $F_{A B}\left(p_{A}, p_{B}\right)$ is the probability that the actual values $v_{A}$ and $v_{B}$ are both smaller than or equal to $p_{A}$ and $p_{B}$. To choose $p_{A}$ and $p_{B}$ are in general no longer separate problems from the perspective of player $S$, but the choice problems become interdependent.

We first consider the case with positive correlation for a given $\beta$. Given

\footnotetext{
${ }^{7}$ We depart from this assumption allowing $A$ and $B$ to observe each other's valuation and the price offer at the end of this section. The equilibrium we find under mutual unobservability is robust to this change in the information assumptions.

${ }^{8} \mathrm{It}$ is easy to confirm that there is a second type of perfect Bayesian equilibrium, in which $A$ and $B$ always reject all offers. Rejecting is mutually optimal for $A$ and $B$ in the continuation game: as none of the players has any influence on the outcome given that the other player rejects the offer, "reject" yields the same payoff as "accept". In turn, if $S$ anticipates this behavior, $S$ may choose any offer price. We do not consider this type of equilibrium in what follows, because it is not robust to refinements. For instance, if the accept/reject decisions of $A$ and $B$ are perturbed, or if $A$ and $B$ choose sequentially, this equilibrium disappears, whereas the equilibrium which we consider further is robust with respect to such refinements.
} 
the symmetry of the problem and the nature of the possible correlation, the optimal choice of prices is symmetric, $p_{A}=p_{B} \equiv p$. In the case of stochastic independence $(\beta=0)$ we have $F_{A B}(p, p)=p p$. In the full correlation case we have $F_{A B}(p, p)=p$. This yields a payoff function equal to

$$
\pi_{S}(p)=2 p(1-2 p+(1-\beta) p p+\beta p) .
$$

Maximization of this payoff yields

$$
p(\beta)=\frac{1}{6(1-\beta)}\left(4-2 \beta-2 \sqrt{1-\beta+\beta^{2}}\right) .
$$

This function has a positive slope on the whole interval of feasible $\beta \in[0,1)$. The optimal price offer by player $S$ is highest for $\beta \rightarrow 1$, and is lower as the valuations of the two players become less stochastically correlated. The payoffs for $A$ and $B$ are

$$
\pi_{A}=\pi_{B}=(1-\beta) \frac{(1-p)^{3}}{2}+\beta \frac{(1-p)^{2}}{2} .
$$

Inserting the price (7) into (8) yields the equilibrium payoffs that will be compared with the other regimes in section 6 .

Intuitively, the player with the lower valuation becomes crucial for whether trade occurs or not. Tying their acceptance decisions to each other gives them some commitment power. Reconsider Figure 1. In the weak-ties regime, for $p_{A}=p_{B}=0.5$, trade occurs only for combinations of $\left(v_{A}, v_{B}\right)$ in the area $a b$. Where one of the two possible deals take place in the standalone regime for the areas $a$ and $b$, player $S$ loses the gains from trade in the weak-ties regime in these cases. Anticipating this higher requirement for trade to take place, player $S$ lowers the aspirations and offers the two items for a lower price. Commitment on weak ties therefore induces a lower price offer, and this is beneficial for the two coalition players. On the negative side, this commitment yields situations such as combinations as in $a$ or in $b$ in which one player would like to accept the offer made to him but cannot, because the offer price is higher than the other coalition player's valuation. 
Commitment causes an additional inefficiency in these cases. Whether negotiating as a weak-ties coalition is advantageous for players $A$ and $B$ will be considered in a later section.

Turning to the case in which the valuations are either stochastically independent or negatively correlated with probabilities $(1-\beta)$ and $\beta$, we can rewrite the payoff function of player $S$ as

$$
2 p[1-2 p+(1-\beta) p p+\beta[\max \{0,(1-2 p)\}]]
$$

Maximization of this payoff for the (relevant) range $p \in[0,1 / 2]$ yields

$$
p(\beta)=\frac{1}{3(1-\beta)}\left(2+2 \beta-\sqrt{1+8 \beta+7 \beta^{2}}\right) .
$$

This function is monotonically decreasing in $\beta$ for $\beta \in[0,1)$. Hence, the price chosen by player $S$ is highest for $\beta=0$, i.e., if the coalition members' valuations are stochastically independent. The payoff of players $A$ and $B$ becomes

$$
\pi_{A}=\pi_{B}=(1-\beta) \frac{(1-p)^{3}}{2}+\beta \frac{(1-2 p)^{2}}{2} .
$$

Inserting (10) into (11) yields the expected equilibrium payoff for $A$ and $B$.

We summarize the main qualitative results as follows:

Proposition 1 In the case with partial positive correlation, the lower the correlation between the coalition members' valuations, the lower the prices offered to the weak coalition. In the case with partially negative correlation, the stronger the negative correlation, the lower the prices offered to the weakties coalition.

Before completing the analysis of the weak-ties coalition, we briefly discuss why the equilibrium underlying Proposition 1 is robust to changes in our information assumptions. Suppose that $A$ and $B$ know the valuations $v_{A}$ and $v_{B}$ of both players and also learn about both offers $p_{A}$ and $p_{B}$. Consider player $i$ 's optimal strategy for given $\left(v_{A}, v_{B}, p_{A}, p_{B}\right)$. If the other player $j$ chooses to reject, then $i$ 's choice is payoff irrelevant. If $j$ accepts, then $i$ is pivotal. The strategy to articulate "accept" if only if $p_{i} \leq v_{i}$ maximizes $i$ 's 
payoff in this case. This is the same optimal decision rule as in the case in which $i$ can observe only $v_{i}$ and $p_{i}$. In turn, if the reactions of $A$ and $B$ to a choice of offers $\left(p_{A}, p_{B}\right)$ are the same as in the case in which each player $i$ can observe only $p_{i}$ and $v_{i}$, then the choice of $p_{A}$ and $p_{B}$ by player $S$ follows the same logic, leading to the same equilibrium choices as in the equilibrium for weak alliances that is underlying Proposition 1. This shows that our analysis does not depend on the particular information assumption we make.

\section{A strong-ties coalition}

A coalition with strong ties provides the players who are members of this coalition with a joint payoff function. Maximizing this joint payoff, they jointly accept the offer by player $S$ if $p_{A}+p_{B}=p_{A B} \leq v_{A}+v_{B}$, otherwise they reject the offer. ${ }^{9}$ The probability for acceptance is, hence,

$$
Q\left(p_{A B}\right) \equiv \operatorname{prob}\left(v_{A}+v_{B} \geq p_{A B}\right)=\int_{0}^{1} \operatorname{prob}\left(v_{B} \geq p_{A B}-x \mid v_{A}=x\right) Z^{\prime}(x) d x
$$

where the $\operatorname{prob}\left(v_{B} \geq p_{A B}-x\right)$ is determined by the joint distribution of $v_{A}$ and $v_{B}$, and $Z(x)$ is the marginal distribution for $v_{A}$. Player $S$ maximizes $p Q(p)$, and the payoff maximizing sum of prices, $p_{A B}$, generally depends on the joint distribution $F_{A B}\left(v_{A}, v_{B}\right)$. For the case of independence, which occurs with probability $(1-\beta)$, the distribution of $v_{A}+v_{B}$ is triangular with support $[0,2]$ and symmetric around 1 . This makes the probability that $v_{A}+v_{B} \geq p_{A B}$ equal to

$$
\operatorname{prob}\left(v_{A}+v_{B} \geq p_{A B}\right)=\left\{\begin{array}{ccc}
1-\left(p_{A B}^{2} / 2\right) & \text { for } & p_{A B} \in(0,1) \\
\left(2-p_{A B}\right)^{2} / 2 & \text { for } & p_{A B} \in(1,2) .
\end{array}\right.
$$

We now consider positive and negative correlation consecutively.

\footnotetext{
${ }^{9}$ Like in the weak-ties regime, there is a second, non-robust equilibrium in which both alliance players reject all offers because they think that the other player is about to reject any offer, so their own decision is inconsequential for the decision outcome.
} 
The case of positive correlation is a linear combination of stochastic independence and perfect positive correlation. Anticipating that the solution to the problem is in the range $p_{A B} \in[0,1]$, the objective function of player $S$ for a given $\beta$ can be written as

$$
\pi_{S}\left(p_{A B}\right)=(1-\beta) p_{A B}\left(1-\left(p_{A B}^{2} / 2\right)\right)+\beta p_{A B}\left(1-\left(p_{A B} / 2\right)\right) .
$$

The first term in this payoff function considers the case in which the valuations are stochastically independent, which has a probability weight of $(1-\beta)$. For stochastically independent valuations, player $S$ earns the price $p_{A B}$ with a probability $\left(1-\left(p_{A B}^{2} / 2\right)\right)$. The second term refers to the case of perfect positive correlation which is relevant with probability $\beta$. For perfect positive correlation player $S$ earns the price $p_{A B}$ with the probability that $v_{A}+v_{B} \geq p_{A B}$, which is equal to $1-\left(p_{A B} / 2\right)$. The function (12) takes on a maximum at

$$
p_{A B}(\beta)=\frac{1}{3(1-\beta)}\left(\sqrt{\beta^{2}+6-6 \beta}-\beta\right)
$$

for given $\beta$ with $\beta \in[0,1)$. The function $p_{A B}(\beta)$ is monotonically increasing in $\beta$. The payoff maximizing price is higher if the valuations $v_{A}$ and $v_{B}$ are more correlated. The joint expected payoff of $A$ and $B$ for a given $p_{A B}$ in the case of stochastic independence is

$$
\int_{p_{A B}}^{1} V\left(V-p_{A B}\right) d V+\int_{1}^{2}(2-V)\left(V-p_{A B}\right) d V=1+\frac{1}{6} p_{A B}^{3}-p_{A B}
$$

where $V \equiv v_{A}+v_{B}$. The joint expected payoff of $A$ and $B$ in the case of perfect correlation is $\left(1-\frac{p_{A B}}{2}\right)\left(1-\frac{p_{A B}}{2}\right)$. The expected payoff for each of the two coalition players as a function of $p_{A B}$ and $\beta$ then is

$$
\pi_{A}=\pi_{B}=\frac{1}{2}\left[(1-\beta)\left(1+\frac{1}{6} p_{A B}^{3}-p_{A B}\right)+\beta\left(1-\frac{p_{A B}}{2}\right)\left(1-\frac{p_{A B}}{2}\right)\right] .
$$

Inserting $p_{A B}$ from (13) into (15) yields the sum of these equilibrium payoffs of $A$ and $B$ as a function of $\beta$ and $p_{A B}$ that will be used in Section 6 when comparing regimes. 
Turn now to the case with stochastic independence with probability (1$\beta$ ) and perfect negative correlation with probability $\beta \in[0,1)$. The payoffs as a function of $p_{A B}$ for the case of independence have been calculated already. For the case of negative correlation, the probability that $v_{A}+v_{B} \geq p_{A B}$ is equal to 1 for $p_{A B} \leq 1$ and equal to zero for $p_{A B}>1$. The expected payoff of player $S$ is

$$
\pi_{S}\left(p_{A B}\right)=(1-\beta) p_{A B}\left(1-\left(p_{A B}^{2} / 2\right)\right)+\beta p_{A B}
$$

for $p_{A B} \in[0,1]$. Maximization of (16) yields

$$
p_{A B}=\min \left\{\frac{\sqrt{6}}{3 \sqrt{(1-\beta)}}, 1\right\} .
$$

The equilibrium payoff for $S$ is $\pi_{S}\left(p_{A B}\right)=\frac{2}{9} \sqrt{6} / \sqrt{1-\beta}$, which is increasing in $\beta$ and takes a maximum of 1 for $\beta \rightarrow 1$.

The expected equilibrium payoff of each of the players $A$ and $B$ is

$$
\pi_{A}=\pi_{B}=\frac{1}{2}\left[(1-\beta)\left(1+\frac{1}{6} p_{A B}^{3}-p_{A B}\right)+\beta\left(1-p_{A B}\right)\right]
$$

for $p_{A B} \in(0,1)$. Using the price (17) that maximizes the payoff of player $S$, for $\beta \in[0,(1 / 3))$ this yields the equilibrium payoffs to be used for a comparison of regimes in Section 6. Summarizing, we find

Proposition 2 In the case of a coalition with strong ties, the price offered is lower the more stochastically independent the valuations of players $A$ and $B$ are. Their payoffs are decreasing in $\beta$ for both positive and negative correlation.

\section{Comparing regimes}

We can now compare the asking prices and equilibrium payoffs in the different regimes for the same values of $\beta \in[0,1)$. Before turning to a comparison of the algebraic results, we consider diagrammatically why player $S$ has an incentive to choose lower asking prices in the two coalition regimes compared 
to the equilibrium prices in the stand-alone regime. We focus on the case of stochastic independence $(\beta=0)$.

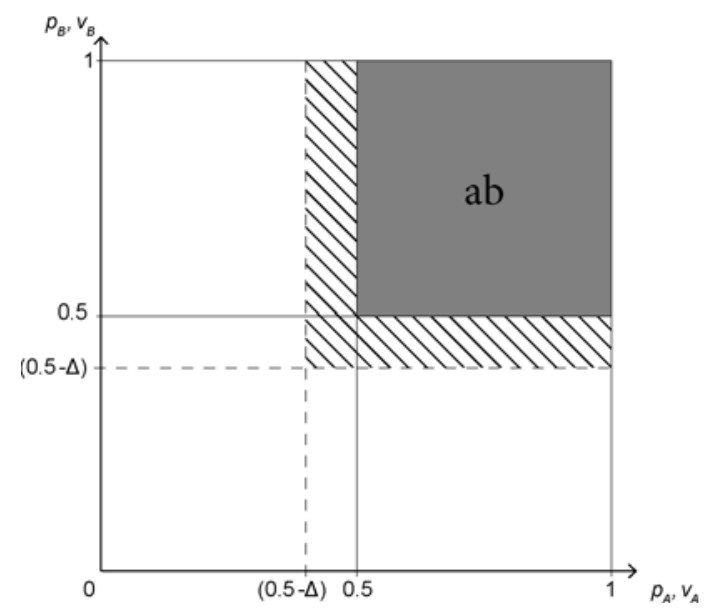

Figure 2a

For the asking price $p_{A}=p_{B}=1 / 2$ both players accept and trade takes place if $\left(v_{A}, v_{B}\right)$ is inside the area ab.

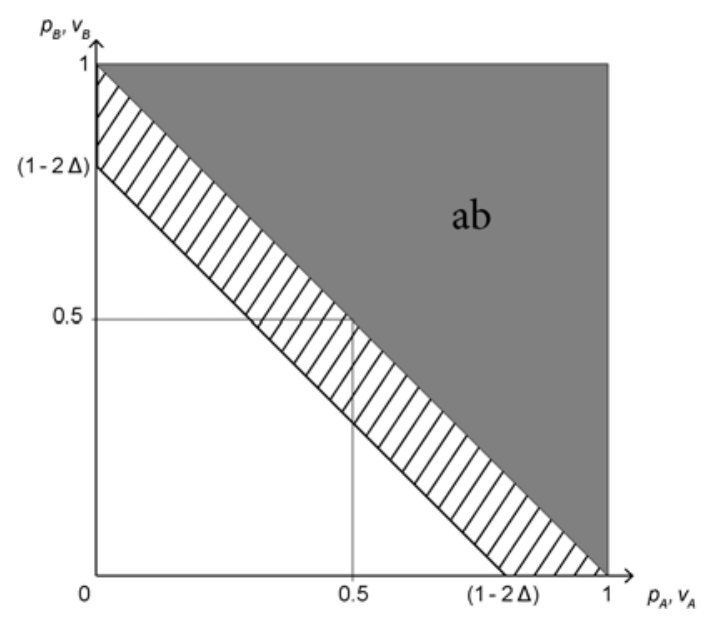

Figure $2 \mathrm{~b}$

For $p_{A}=p_{B}=1 / 2$ both players accept and trade takes place if $\left(v_{A}, v_{B}\right)$ is inside the area ab.

The equilibrium for the stand-alone regime for $\beta=0$ is mapped in Figure 1 by $p_{A}=p_{B}=1 / 2$. It is also the starting point for a discussion of player $S$ 's incentives in Figure 2a. For the weak-ties regime, at prices $p_{A}=p_{B}=0.5$ trade takes place with probability corresponding to the area $a b$, and if it takes place, it takes place with both players. The expected number of units traded is therefore $2(a b)=0.5$. This smaller expected number of trades compared to $a+b+2(a b)$ in the stand-alone regime means that the 'price effect' of a reduction in prices is smaller at $p_{A}=p_{B}=0.5: S$ loses revenue from a reduction in prices on a smaller number of trades in this regime than in the stand-alone regime. Moreover, if $S$ reduces the prices at 0.5 by $\Delta$, the increase in probability that trade takes place with both players is equal the shaded area ('quantity effect'). This quantity effect affects two units of trade, one with $A$ and one with $B$. Hence, the increase in expected quantity traded at $p_{A}=p_{B}=0.5$ is equal to $2 \Delta$, exactly the same as in the stand-alone regime. In the stand-alone regime the price effect and the quantity effect just balanced each other at $p_{A}=p_{B}=1 / 2$. In the weak-ties 
regime the quantity effect is the same, but the price effect is smaller. This explains why $S$ prefers to set a lower price in the weak-ties regime than in the stand-alone regime.

For the strong-ties regime consider Figure 2b. Starting the discussion again at $p_{A}=p_{B}=0.5$, trade of two units takes place for all combinations of valuations in the area to the upper-right of the line from the upper-left corner $(1,0)$ to the lower-right corner $(1,0)$. In expectation, 1 unit is traded at these prices, which is the same expected number of trades as for the standalone regime. A reduction in both prices by $\Delta$ starting from $p_{A}=p_{B}=0.5$ generates a reduction of $p_{A B}=p_{A}+p_{B}$ of size $2 \Delta$. For small $\Delta$, this increases the expected number of trades by $4 \Delta$, and this increase is larger than for the stand-alone regime. For the strong-ties regime, the price effect is the same as in the stand-alone regime, but the quantity effect is larger. This explains why $S$ has an incentive to choose lower prices in the strong-ties regime than in the stand-alone regime.

We now turn to the algebraic results in the previous sections and use them to compare equilibrium prices more formally.

Prices Figures $3 \mathrm{a}$ and $3 \mathrm{~b}$ show the equilibrium prices for the three regimes (solid line: stand-alone regime; dashed line: weak ties; dotted line: strong ties). Both types of coalition have a strategic effect on the asking price chosen by player $S$. The price reduction is largest if the valuations $v_{A}$ and $v_{B}$ are stochastically independent and weakens as positive correlation increases. The effects differ quantitatively for positive correlation (Figure 3a) and negative correlation (Figure 3b). 


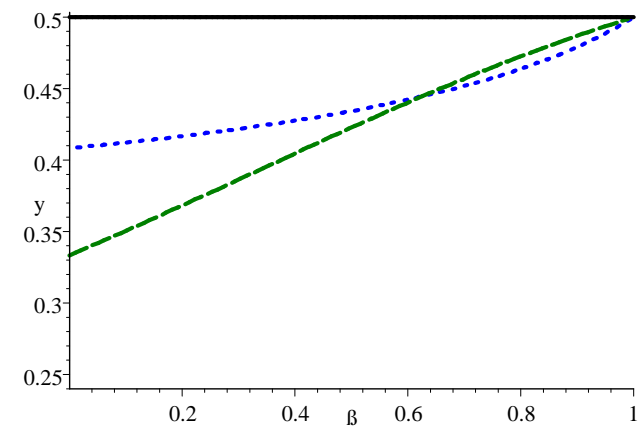

Figure 3a

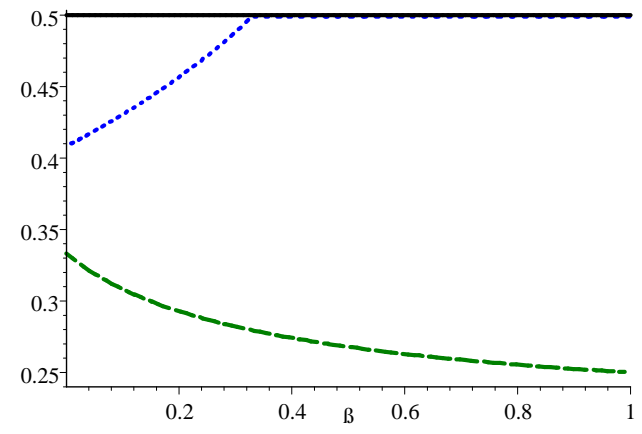

Figure 3b

Equilibrium asking price for the case of positive correlation (Figure 3a) and negative correlation (Figure 3b). Black (solid): stand-alone; green (dashed): weak-ties; blue (dotted): strong-ties.

Consider the intuition for the weak-ties regime. If players $A$ and $B$ are more likely to have similar valuations (positive correlation), then the fact that their agreements are tied to each other by weak ties has a smaller impact compared to making separate offers to each of them. The risk is smaller that trade with $A$ that would have taken place in the stand-alone regime at some prevailing price is prevented by a low valuation of $B$. For perfect positive correlation, this risk completely disappears. For negative correlation, from the information perspective of player $S$ who does not know the actual values $v_{A}$ and $v_{B}$, the player must attribute a large likelihood to that actual valuations of $A$ and $B$ are very different. This makes $S$ more cautious and reduces the asking prices in the weak-ties regime.

For strong ties, due to side payments, the comparison that decides about acceptance or rejection is whether $p_{A B} \leq v_{A}+v_{B}$. When $S$ makes an offer, $S$ does not know the actual values $v_{A}+v_{B}$, but maximizes an expected payoff using the distribution from which the actual values $v_{A}+v_{B}$ are drawn. For negative correlation, the distribution of $v_{A}+v_{B}$ shows less dispersion, making its value more predictable for $S$. This is most evident for the case of perfect negative correlation, in which case $v_{A}+v_{B}$ is equal to 1 with probability 1 . All uncertainty about the coalition players' joint willingness 
to pay disappears in this case. Player $S$ can then set $p_{A B}=1$ and extract all rents from the coalition players. This effect is weaker, but also at work if the distribution $F_{A B}$ exhibits some, but not perfect negative correlation: this reduces the uncertainty that player $S$ faces and tends to reduce the information rent which players $A$ and $B$ enjoy in the equilibrium.

Payoffs This type of logic can also be used to explain the results when considering the equilibrium payoffs of players $A$ and $B$. Figures $4 \mathrm{a}$ and $4 \mathrm{~b}$ show these payoffs for positive correlation (left) and for negative correlation (right).

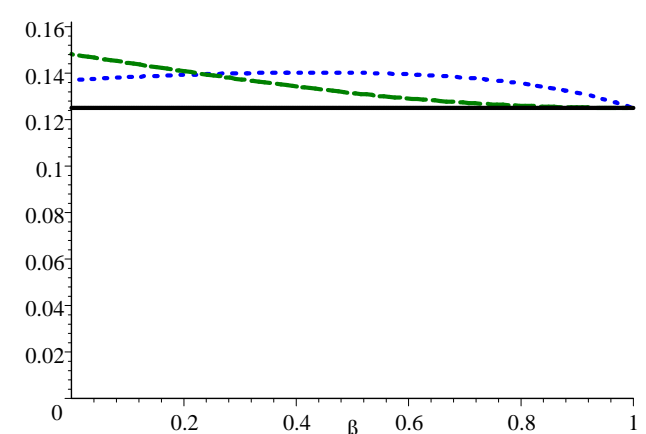

Figure 4a

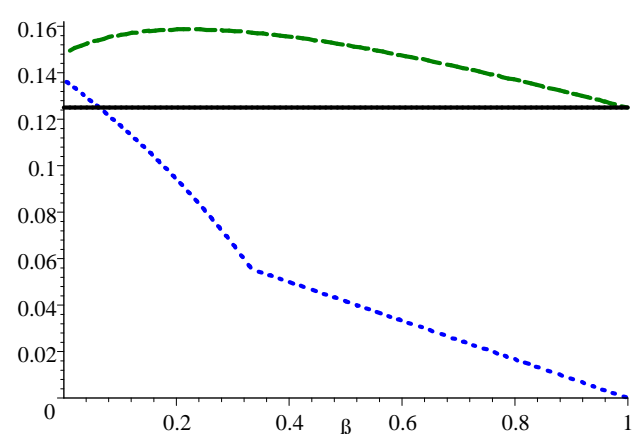

Figure $4 b$

Equilibrium payoffs for coalition members for positive correlation (Figure 4a) and negative correlation (Figure 4b). Black (solid): stand-alone; green (dashed): weak-ties; blue (dotted): strong-ties.

Compare first the coalition with weak ties with the benchmark case. For the full range of positive correlation $\beta \in[0,1)$ the coalition players' payoff exceeds their payoff in the benchmark case. Only in the case of perfect positive correlation $(\beta \rightarrow 1)$ is the payoff not different from the benchmark payoff. Intuitively, the strategic effect on the asking price is stronger than the inefficiency emerging from the unanimity requirement. As $\beta \rightarrow 1$ unanimity does not impose an additional requirement, leading to the same asking price per player in the regime with a coalition with weak ties. For negative correlation there is a similar intuition. Negative correlation makes the unanimity requirement a very strong condition and yields strong commitment. However, 
this unanimity also causes inefficiency. Still, unless the negative correlation is perfect, the commitment effect on prices for the payoff of $A$ and $B$ is stronger than the payoff loss for $A$ and $B$ that is caused by the inefficiency.

Turn now to a comparison with the strong-ties coalition. Unanimity also has the potential to make player $S$ more cautious regarding the price charged in this regime, compared to the stand-alone regime. On the other hand, due to the summing up and splitting the surplus from any deal that is made, one coalition player does not prevent the other coalition player from making a very valuable deal, provided that the two deals together generate a joint surplus to them. The focus on joint surplus induces the coalition to purchase both items even if the valuation for one of the items is small in comparison to the total asking price, provided that the second item is sufficiently highly valued. The seller need not be too afraid of charging a high price in total, as the higher valuation for one item may compensate the low valuation of the second item that might prevail. However, there is still some inefficiency from the fact that both items can only be traded jointly, such that a very low valuation for one item may jeopardize the trade in the second item even if this has a very high valuation.

Overall, in the case of stochastic independence, the average price per item charged in the strong coalition regime is still lower than in the standalone regime. This yields a higher payoff for the coalition players, compared to the stand-alone outcomes. This joint effect also holds for moderate positive or negative correlation. If the valuations of the coalition players become strongly positively correlated and eventually identical, at that point commitment from potentially different valuations vanishes and the problem degenerates to one that is equivalent to the situation where player $S$ makes an offer to one single stand-alone player. This is why the payoff in this regime returns to the payoff in the benchmark case for positive correlation with $\beta \rightarrow 1$. For negative correlation, players $A$ and $B$ suffer from a reduction in uncertainty as regards the sum of their valuations in the strong-ties coalition. Uncertainty about their joint valuation generates an information rent 
to them. With the disappearance of this uncertainty, the information rent attached to it also melts away. This is why the payoff of coalition players sharply declines as the negative correlation becomes large.

One can also look at a comparison between the two coalition regimes. None of the regimes globally dominates the other. The strategic price effect in the case of weak ties is more robust to changes in the joint distribution of valuations than in the case of strong ties, but there are joint distributions $F_{A B}$ for which the payoff of the coalition is higher with strong ties than with weak ties.

Efficiency The three regimes can also compared with respect to efficiency, defined as the expected sum of the payoffs of all three players. The efficiency benchmark is the situation in which no rent is lost. This happens if trade always takes place. The sum of rents in this case is equal to $E\left(v_{A}+v_{B}\right)=1$ in expectation. For the stand-alone regime, the expected rent is equal to the sum of the equilibrium payoffs for players $A, B$, and $S$, and, using (4) these sum up to $3 / 4$ (the solid line in Figures $5 \mathrm{a}$ and $5 \mathrm{~b}$ ). We can also use the respective equilibrium prices and payoffs and sum up payoffs of all three players for the weak-ties regime and for the strong-ties regime, for positive and negative correlation. Plotting these rents yields Figures 5a (positive correlation) and 5b (negative correlation). These show that the strong-ties case (dotted lines) has the best efficiency properties, and that efficiency is lowest in the weak-ties regime (broken lines). Full efficiency is reached in the strong-ties regime with perfect negative correlation; private information disappears in this case, but this is to the disadvantage of the coalition of players $A$ and $B$ who lose all rent in this regime at this point. 


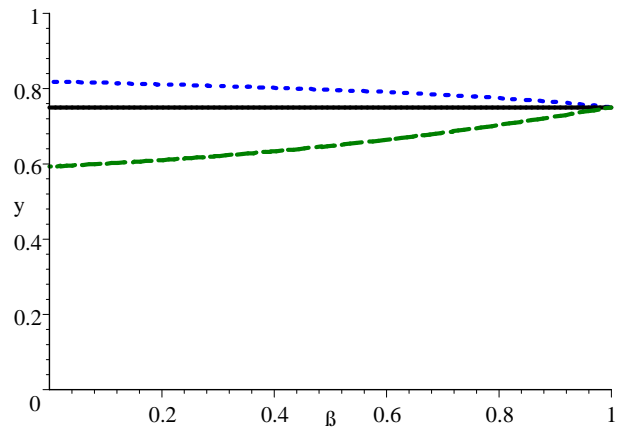

Figure 5a

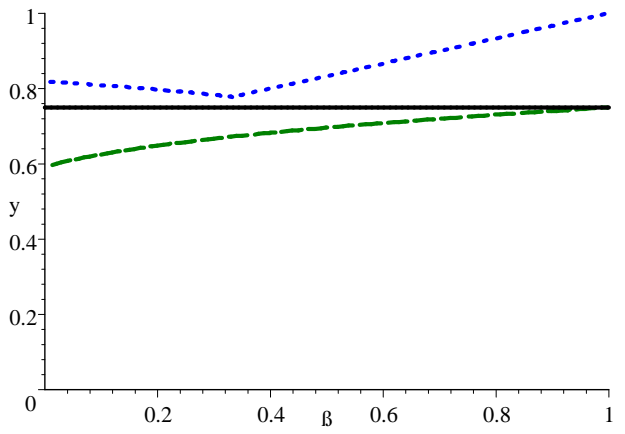

Figure 5b

Efficiency relative to full efficiency for the three regimes for positive correlation (Figure 5a) and negative correlation (Figure 5b).

Black (solid): stand-alone; green (dashed): weak-ties; blue (dotted): strong-ties.

Endogenous regimes? The analysis of equilibrium payoffs suggests that joint negotiations in a coalition provide strategic advantages for a broad range of parameters, compared to stand-alone negotiations. We have considered and compared exogenously given regimes. But where coalitions are voluntarily formed, our results suggest that coalitions should be more frequently observed than without this strategic advantage.

We would not conclude from this that coalitions should be ubiquitous, however. Too many other aspects may play a role if coalitions are endogenous. First, the decision whether to form a coalition and with whom may reveal or signal certain types of information to players outside the coalition. This is not a problem if the decision to form a coalition takes place before $A$ and $B$ learn about their true valuations. In this case no information is revealed because the players make their coalition choices while they are still uninformed themselves. But for many situations they may already have some private information at the point of time when a coalition could be formed. In our context, the incentive for $A$ and $B$ to enter into a coalition depends on their actual valuations; accordingly, if these are, or could be known prior to such decisions, they would signal information and affect the 
price offers (Wagner 2004). Second, the formation of a coalition - or abstaining from it - may convey or signal information to non-coalition players that may be relevant in other contexts. ${ }^{10}$ Third, coming back to negotiations by the European Union and its member states with non-member states or other groups: acting as a coalition in negotiations with tax havens for information exchange agreements or with other countries for double taxation treaties may yield strategic advantages. However, this would compromise the principles according to which competence on tax policy is strictly on the nation level and not at the European level. And member countries may not want to sacrifice their national sovereignty on tax policy for the strategic advantages in negotiating international tax conventions. Fourth, bargaining as a coalition requires the ability to commit. If one of the members of a coalition prefers to accept his offer, but the other does not, this member is tempted to default and to accept the offer anyway. In a many institutional frameworks a sufficient degree of commitment may not be feasible. ${ }^{11}$ Fifth, coalition formation is a dynamic process which is sensitive to what happens if some player joins or departs from a given coalition. ${ }^{12}$

\footnotetext{
${ }^{10}$ Roy (1994) and Sagan and Suri (2003) offer examples for such signals that may serve to build reputation and may allow to make threats credible that would not be credible among purely rational players.

${ }^{11}$ It should be noted that since Thuycidides and later Machiavelli, many analysts have been skeptical, for instance, of the war-time reliability of alliance partners to live up to their commitments. A more recent example of this is to be seen in Schmidt's (1953) discussion of the phrase "Perfidious Albion." Indeed, early quantitative work in political science (Sabrosky, 1980) concluded that the failure rate was about 75 percent. A modest one in four chance of an ally living up to its commitments is not a very reassuring situation in a violent, anarchic world. More recent and detailed empirical work (Leeds, Long and Mitchell, 2000 and Leeds, 2003) show, however, that the level of reliability is far higher and, as a consequence, the failure to keep commitments likely only 25 percent of the time. Importantly, these failures are often the product of changes since the commitment was made that have reduced the costs of failing to keep the commitment or changes that have transpired within the alliance.

${ }^{12}$ Coalition formation and its evolution is typically a dynamic process. For a study of some of several aspects with several types of group interaction see Ray and Vohra (2001),
} 
Our analysis also shows that the strategic advantage of a coalition is higher for strong-ties coalitions compared to weak-ties coalitions, at least for a broad parameter range. Strong-ties coalitions, however, require sidepayments ("transferable utility"). As discussed also in the context of the EU example, the frontiers between weak and strong ties are difficult to draw, as side payments may occur with a delay, and in a larger cooperative context. Generally, contracts on side-payments may be costly to implement, to execute and to enforce.

Another aspect that makes an empirical test of the theory difficult is the conceptual difference between countries' symmetry ex-ante (the type distribution from which $v_{i}$ are drawn) and potential symmetry or asymmetry ex-post (the actual values of $v_{i}$ ). Unless coalitions negotiate repeatedly in a context that is time invariant, the correlation between the reservation prices of members of a coalition is difficult to assess. All this, plus historical and institutional idiosyncrasies make it difficult to develop an empirical strategy. However, the problem can be suitably studied in laboratory experiments and clear cut predictions can be tested more systematically. This is something we would like to explore in the future.

\section{Conclusions}

This paper examines the role of coalitions in bargaining problems with onesided incomplete information in which one uninformed player can offer two informed players one item each. This uninformed player is the proposer and makes take-it-or-leave-it offers. The two informed players are the responders. We study two types of coalitions between two responders and compare the coalition outcomes with separate take-it-or-leave-it offers to each of the informed players. Coalitions are understood here as commitment devices that allow players to make their acceptance decision for the offers they re-

Konishi and Ray (2003) and Hyndman and Ray (2007). 
ceive in a joint decision fashion. We compare how a coalition between the informed responders and its internal governance structure affects the price offers made by the uninformed player. If the responders are joined in coalition, this typically reduces the price which the uninformed player bids. This is the strategic price effect for the responders being in a coalition. The coalitions also has an efficiency effect. Since it turns separate decisions into joint decisions, it may cause joint rejection where, given the asking prices, players may jointly prefer one of them to accept the offer made, whereas the other player is better-off not accepting the offer. While there is no general conclusion regarding whether acting as a coalition is superior to a stand-alone regime, and, in which case, what type of coalition should be chosen, the analysis reveals strong strategic effects of coalition formation, strengthening the coalition's power in negotiations with an uninformed outsider.

\section{References}

[1] Bearce, David H., Kristen M. Flanagan, and Katharine M. Floros, 2006, Alliances, internal information, and military conflict among member-states, International Organization, 60(3), 595-625.

[2] Bond, Philip, and Hülya Eraslan, 2010, Strategic voting over strategic proposals, Review of Economic Studies, 77, 459-490.

[3] Chatterjee, Kalyan, and William Samuelson, 1983, Bargaining under incomplete information, Operations Research, 31(5), 835-851.

[4] Eppstein, Gloria, Sina Gerlach, and Maren Huser, 2010, The EU's impact in international climate change negotiations - the case of Copenhagen, unpublished manuscript.

[5] Feddersen, Timothy, and Wolfgang Pesendorfer, 1998, Convicting the innocent: The inferiority of unanimous jury verdicts under strategic voting, American Political Science Review, 92(1), 23-35.

[6] Groenleer, Martijn L. P., and Louise G. van Schaik, 2007, United we stand? The European Union's international actorness in the cases 
of the international criminal court and the Kyoto protocol, JCMS, 45(5), 969-998.

[7] Harsanyi, John C., and Reinhard Selten, 1972, A generalized Nash solution for two-person bargaining games with incomplete information, Management Science, 18(5), P80-P106.

[8] Hyndman, Kyle, and Debraj Ray, Coalition formation with binding agreements, Review of Economic Studies, 74(4), 1125-11-47.

[9] Jupille, Joseph, 1999, The European Union and international outcomes, International Organization, 53, 409-425.

[10] Konishi, Hideo, and Debraj Ray, 2003, Coalition formation as a dynamic process, Journal of Economic Theory, 110(1), 1-41

[11] Konrad, Kai A., 2012, Information alliances in contests with budget limits, Public Choice, 151(3-4), 679-693.

[12] Kroll, Stephan, and Jason F. Shogren, 2008, Domestic politics and climate change: international public goods in two-level games, Cambridge Review of International Affairs, 21, 563-583.

[13] Leeds, Brett Ashley, 2003, Alliance reliability in times of war: Explaining state decisions to violate treaties, International Organization, $57(4), 801-827$.

[14] Leeds, Brett Ashley, Andrew G. Long, and Sara McLaughlin Mitchell, 2000, Reevaluating alliance reliability: Specific threats, specific promises, Journal of Conflict Resolution, 44(5), 686-689.

[15] Manzini, Paola, and Marco Mariotti, 2005, Alliances and negotiations, Journal of Economic Theory, 121, 128-141.

[16] Manzini, Paola, and Marco Mariotti, 2009, Alliances and negotiations: an incomplete information example, Review of Economic Design, $13,195-203$.

[17] Meunier, Sophie, 2000, What single voice? European institutions and 
EU-U.S. trade negotiations, International Organization, 54(1), 103135 .

[18] Ray, Debraj, and Rajiv Vohra, 2001, Coalitional power and public goods, Journal of Political Economy, 109(6), 1355-1384.

[19] Roy, Denny, 1994, North Korea and the 'madman' theory, Security Dialogue 1994 (25), 307-316.

[20] Sabrosky, Alan, 1980, Interstate alliances: Their reliability and the expansion of war, in: The correlates of war II: Testing some realpolitik models, ed. J. David Singer, New York: Free Press.

[21] Sagan, Scott D., and Jeremi Suri, 2003, The madman nuclear alert: secrecy, signaling, and safety in October 1969, International Security, $27(4), 150-183$.

[22] Scharpf, Fritz W., 1988, The joint-decision trap: lessons from German federalism and European integration, Public Administration, 66(3), 239-278.

[23] Scharpf, Fritz W., 2000, Notes toward a theory of multilevel governing in Europe, Köln: Max-Planck-Institut für Gesellschaftsforschung Discussion Paper 00/5.

[24] Scharpf, Fritz W., 2006, The joint-decision trap revisited, Journal of Common Market Studies, 44(4), 845-864.

[25] Schmidt, H.D., 1953, The idea and slogan of "Perfidious Albion," Journal of the History of Ideas, 14(4), 604-616.

[26] Stasavage, David, 2002, Credible commitment in early modern Europe: North and Weingast revisited, Journal of Law, Economics and Organization, 18(1), 155-186.

[27] Wagner, R. Harrison, 2004, Bargaining, war, and alliances, Conflict Management and Peace Science, 21, 215-231. 